\title{
Low-fidelity Paediatric Surgical Simulation: Description of Models in Low-Resource Settings
}

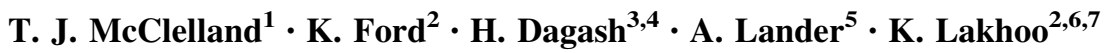

Published online: 31 January 2019

(C) The Author(s) 2019

\begin{abstract}
Background Surgical simulation is an important aspect of competency-based training. Recent trends in paediatric surgical simulations have migrated towards high-fidelity simulation with advanced technology resulting in models which are expensive and largely inaccessible in low- and middle-income countries.

Methods This article describes four wet simulation models of common surgical procedures in paediatric population created with animal tissue from local abattoir. The models are designed to provide a framework for others to make the models and benefit from the training opportunity they provide especially in low-middle-income countries.

Results The models created in the wet laboratory are neonatal bowel anastomosis, duodenoduodenostomy for discrepancy anastomosis, gastrostomy and pyeloplasty. These models are easily reproducible in resource-challenged healthcare setting as they are low cost, utilise locally available resources and require only a basic set of surgical instruments with which to perform the procedures.

Conclusion These models provide locally accessible material for sustainable training programmes which are fundamental in developing safe and affordable surgical care worldwide.
\end{abstract}

K. Lakhoo

kokila.lakhoo@nds.ox.ac.uk

University of Oxford Medical School, Oxford, UK

2 Nuffield Department of Surgery, University of Oxford and Oxford University Hospitals, Oxford, UK

3 Department of Pediatric Surgery, Leicester Royal Infirmary, Leicester, UK

4 Soba University Hospital, Khartoum, Sudan

5 Department of Paediatric Surgery, Birmingham Children's Hospital, Birmingham, UK

6 Muhimbili National Hospital, Dar es Salaam, Tanzania

7 Department of Paediatric Surgery, John Radcliffe Hospital, Oxford OX3 9DU, UK

\section{Introduction}

Surgical simulation is an important aspect of competencybased training characterised by guided experiences replicating aspects of the intra-operative environment to improve basic surgical skills. In recent years, there has been a shift towards high-fidelity simulation involving advanced technology such as virtual reality and 3-D printed models. These methods, however, are expensive and largely inaccessible outside of high-income tertiary centres. Following the 2030 Lancet Commission on Global Surgery meeting, it was acknowledged that provision of sustainable and locally accessible training programmes would be fundamental in improving safe and affordable surgical care in low- and middle-income countries (LMICs) [1]. 
This article describes four wet surgical simulation models: bowel anastomosis; duodenoduodenostomy; gastrostomy; and pyeloplasty. The article aims are threefold: (1) describing how the model materials are sourced, (2) assembly of materials into the simulation model and (3) utilisation of the model for the individual surgical trainee and trainer. Importantly, these simulations are low cost and low fidelity-enabling those in LMICs to acquire the requisite materials with ease and provide training. The models have been trialled and used successfully in nine courses (run by the International Affairs Committee, British Association of Paediatric Surgeons, UK), held in low- and middle-income countries across Africa since 2008. The feedback and outcomes of the courses are beyond the scope of this article and thus not reported.

\section{Materials and methods}

The method of making the models is described individually below. Animal material is easily accessible from a local butcher. The total cost of each model should be less than 10USD. Common to all of the models is a basic set of surgical instruments (needle holder, toothed and nontoothed forceps, stitch scissors, mosquito forceps, sutures) and simulation tools: an operating pad (reinforced sponge covered in a waterproof lining), thumb tacks, syringe and a nasogastric tube.

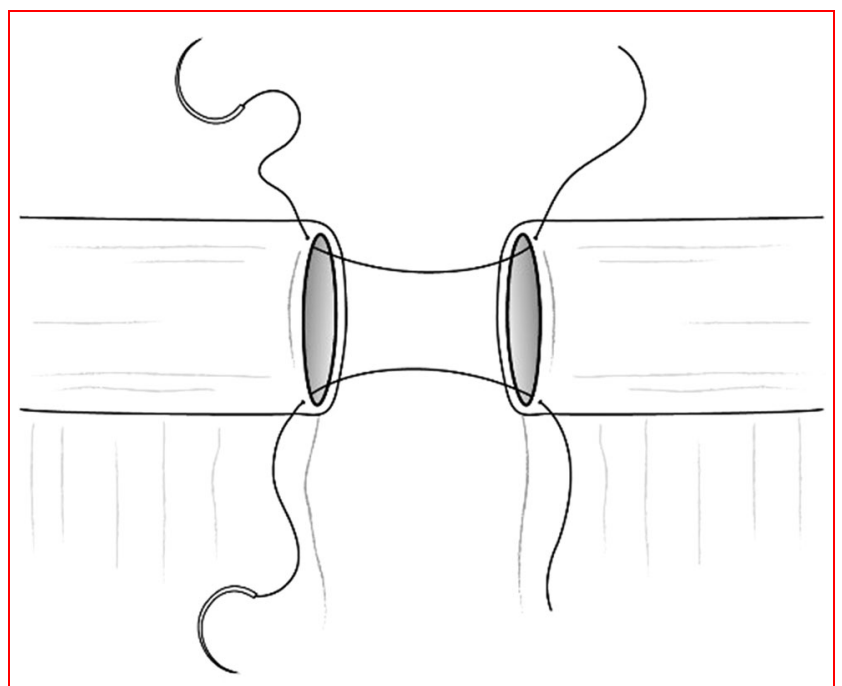

Fig. 1 Diagram of the bowel anastomosis model demonstrating the two segments of sheep intestine. The proposed placement of the $5 / 0$ absorbable sutures to represent a mesenteric (superior) and antimesenteric (inferior) border is shown

\section{Bowel anastomosis}

This model uses a bisected segment of sheep intestine. The two bowel segments are aligned facing each other, and corner stitches are placed using 5/0 absorbable suture. These represent the mesenteric and anti-mesenteric borders. The stitch is placed from outside-into inside-out so the knot is on the outside (Fig. 1). The anterior wall is then closed with a 5/0 absorbable suture using an interrupted seromuscular stitching technique. The corner stitches should now be reversed by passing the mesenteric stitch through the defect in the mesentery and flipping the antimesenteric stitch in the other direction (Fig. 2). The anastomosis is completed by closing the posterior wall (which is now anterior) in the same manner.

It is our preference to test the anastomosis. This is done by occluding the bowel a distance of about $1 \mathrm{~cm}$ from the anastomosis and using a 5-ml syringe with a blue needle, injecting saline into the lumen and testing for a leak.

\section{Duodenoduodenostomy}

Duodenoduodenostomies are used to bypass duodenal atresia or stenosis causing intestinal obstruction. This mock bowel size discrepancy simulation model replicates the approach commonly known as a diamond duodenoduodenostomy.

This model requires half a sheep bladder and a segment of sheep bowel in addition to the basic equipment detailed above. In this simulation, the proximal dilated duodenum is represented by the bladder itself, whilst the bowel loop replicates the post-stenotic/atretic non-dilated distal duodenum.

A transverse incision is made in the bladder. This should be at least one centimetre from the atresia to minimise the risk of damage to surrounding pancreatobiliary structures. A longitudinal incision of the same length is then made in the bowel loop. These incisions are shown schematically in Fig. 3. A feeding tube can be inserted into both 'duodenotomies' to mimic assessment of proximal and distal patency of the tract-this ensures there are no concurrent segments of atresia.

The 3/0 absorbable stays are placed at the ends of both incision margins. Using 5/0 absorbable sutures, the corner stitches are placed first. Drawing the suture from outside to inside in the superior segment and from inside to outside on the corresponding distal segment, i.e. A-A and B-B, a partial diamond anastomosis is formed. An internal suture connecting D-D completes the anastomosis.

\section{Gastrostomy}

Gastrostomies are commonly indicated in paediatric populations when there is a need for long-term feeding, or 


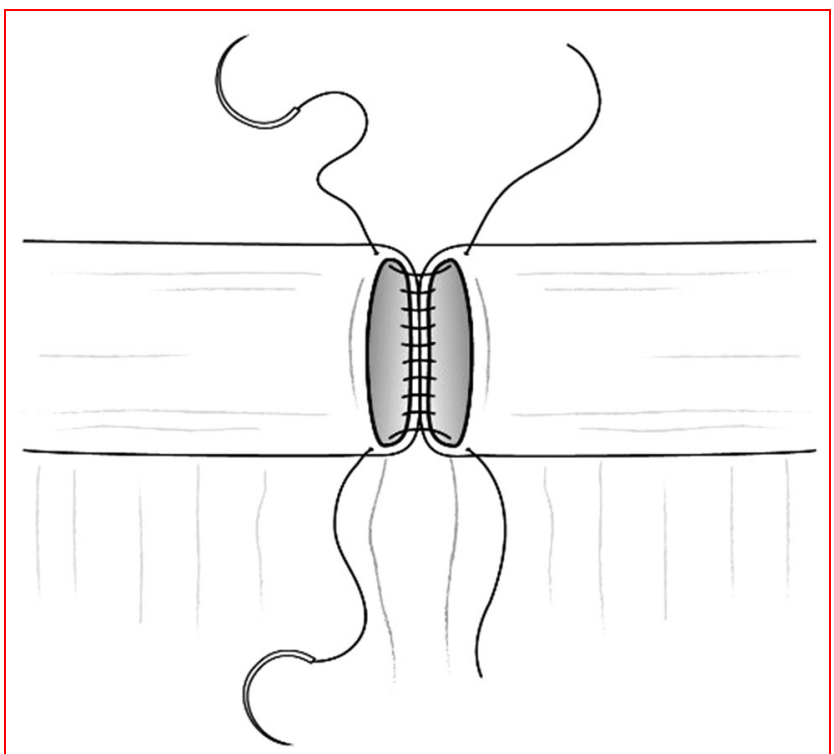

Fig. 2 Diagram demonstrating the closure of the anterior wall with interrupted seromuscular stitches. This should be repeated on the posterior wall to complete primary closure of the anastomosis

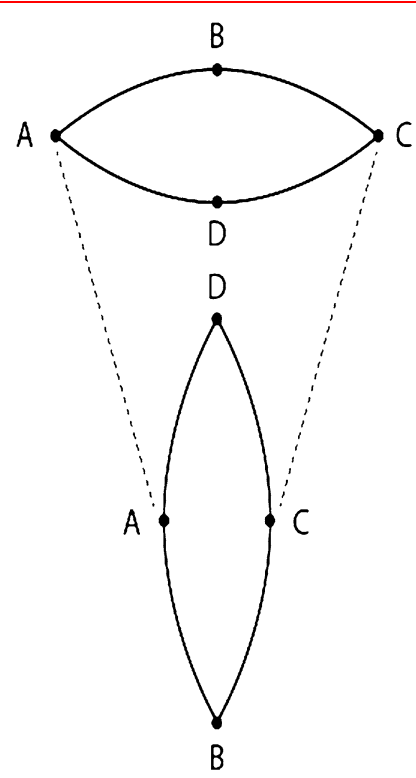

Fig. 3 Schematic representation of the diamond incisions to be made in the proximal bladder (horizontal incision, superiorly) and the distal bowel loop (vertical incision, inferiorly)

decompression. This simulation closely resembles the Stamm-type gastrostomy enabling surgeons to practice two key aspects of gastrostomy placement. This includes: selection of opening site on the anterior gastric wall and creation of a serosa-lined invagination to house a gastrostomy tube using concentric purse-string suturing technique. This model requires a sheep stomach and Foley catheter.
To correctly site a gastrostomy in the anterior gastric wall, the operator must first mark with a pen the ideal location for insertion of the catheter resembling the gastric tube. Starting at the 12 o'clock position, a circumferential $360^{\circ}$ suture is placed around the proposed exit site. A second suture is placed starting at the 6 o'clock position following the first circumferentially (Fig. 4).

An incision is made in the middle of the sutures, and a Foley catheter is placed into the gastric cavity. The sutures should be elevated before creating the incision; this allows better gastric access and prevents potential damage to the posterior wall. The inner purse-string suture is then tied, followed by the outer suture. The balloon is then inflated. Three further $3 / 0$ absorbable sutures anchor the stomach to the anterior abdominal wall.

\section{Pyeloplasty}

Congenital ureteropelvic obstruction may require early pyeloplasty to preserve upper tract function. Here, half a sheep bladder represents the pelvis, and a loop of bowel represents the ureter.

The first step in the pyeloplasty is to mobilise the redundant pelvis and carefully identify and isolate the stenotic ureter (Fig. 5). A 5/0 absorbable suture can be inserted into the proposed upper limit of the renal pelvis incision, allowing manipulation and visualisation of the ureteropelvic junction. The anterior wall of the renal pelvis should be incised to reveal the proximal stenotic ureter. The ureter is then spatulated laterally (Fig. 6).

Anchoring sutures are placed from the tip of the spatulated ureter to the lower end of the pelvis before complete dismembering of the ureteropelvic junction. Another stitch should be placed connecting the medial end of the ureter to the upper end of the pelvis (Fig. 7).

The posterior wall of this anastomosis is performed and a stent is passed (Fig. 8). The anterior wall is then closed and the pelvis closed in a continuous manner. Using the stent, a leak test is then conducted.

\section{Discussion}

\section{Simulation in paediatric surgery}

Paediatric surgery is a diverse specialty where surgeons are required to have a versatile technical skill set. Development of these abilities demands sustained practice and continuous educational input. These facts, coupled with the considerable consultant-led workload and ethical problems with inexperienced 'practice' on the paediatric population, have led to an increase in the need for simulation-based education (SBE). These recreations of surgical 


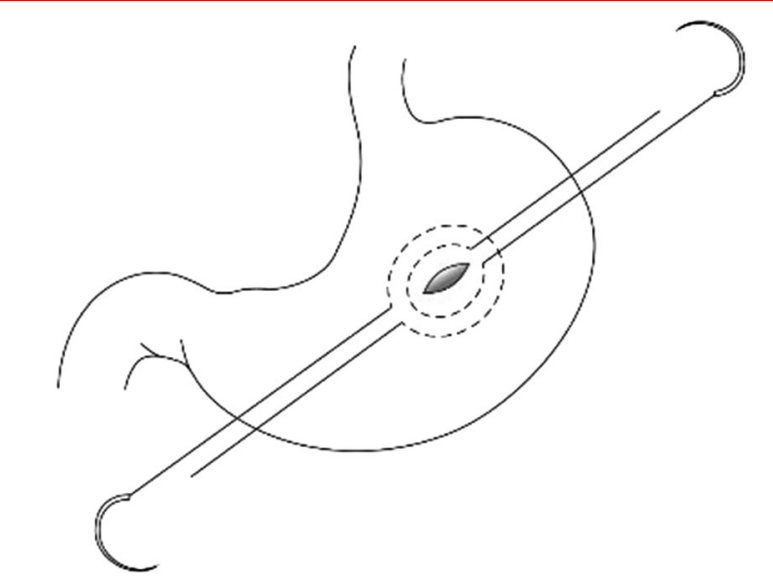

Fig. 4 Diagram of the gastrostomy simulation. The concentric suturing is demonstrated at the body of the stomach. Once placed, an incision can be made and a Foley catheter placed into the gastric cavity of the sheep stomach

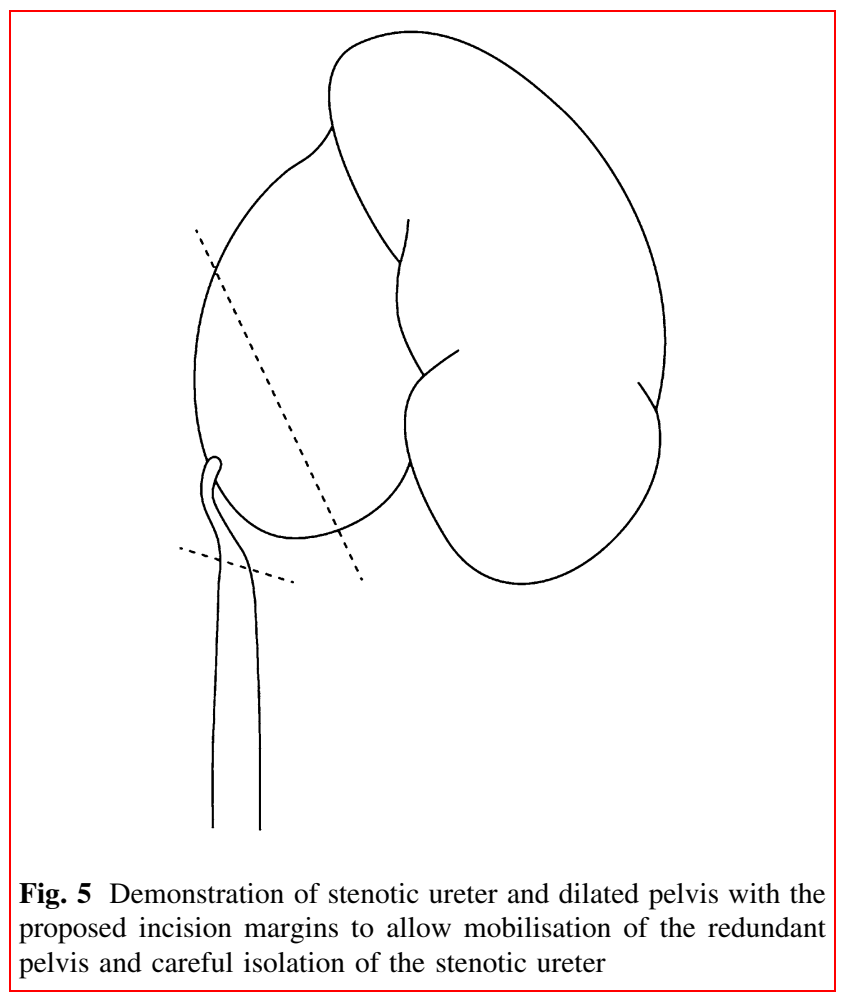

interventions provide a platform for supervised learning and competency-based assessment of trainees, to reduce variability in trainees skills and improve clinical outcomes [2]. These principles of SBE have long been identified and validated in the adult populations [3]; however, authentic and, more importantly, accessible paediatric simulation models are in their infancy.

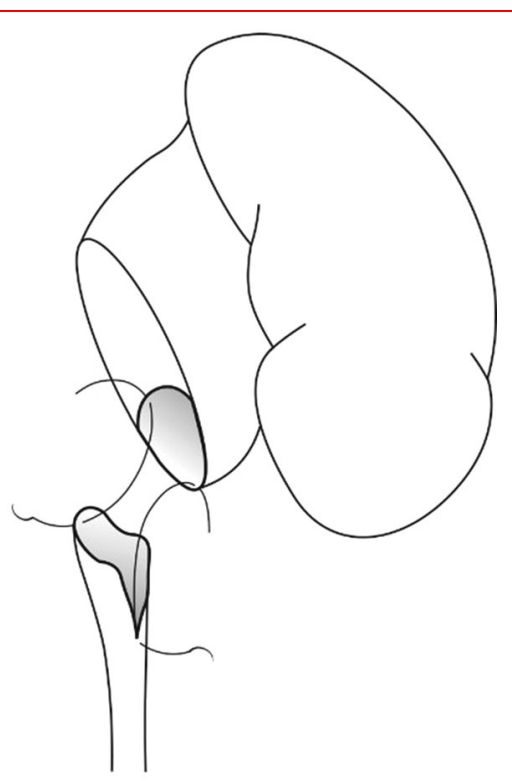

Fig. 6 A post-incision schematic showing the splayed proximal ureter and correct placement of the anchoring sutures

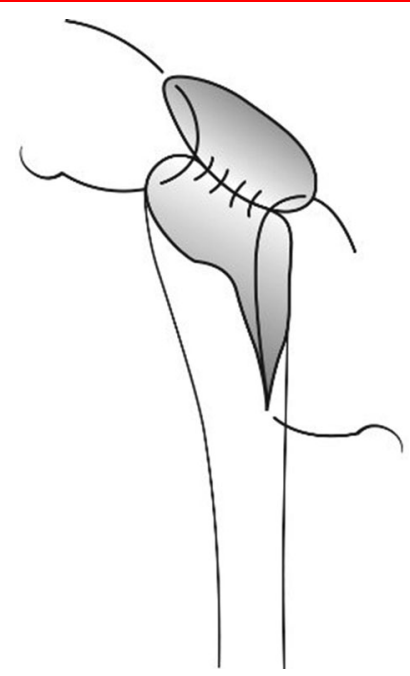

Fig. 7 Formation of primary closure connecting the newly dilated superior aspect of the ureter to the inferior aspect of the incised renal pelvis

\section{Accessibility of simulation}

Current trends in paediatric simulation lean towards highfidelity, and thus high-cost, models. In the advent of 3-D printing and enhanced virtual reality technology, these simulations, although realistic, are largely inaccessible to most healthcare systems. Furthermore, although by definition high-fidelity, ex vivo [4] and in vivo [5] animal 


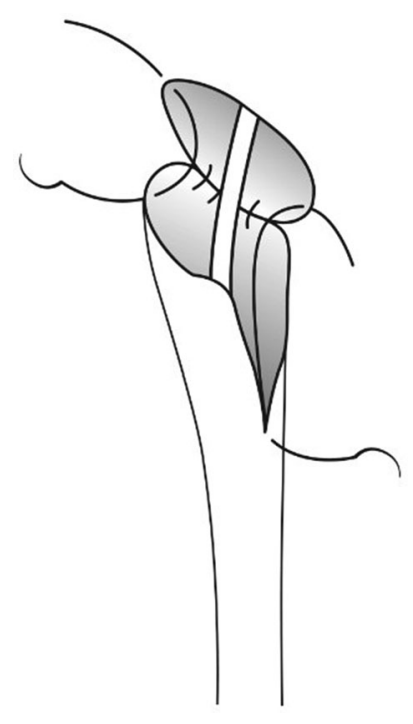

Fig. 8 Placement of a Foley catheter as a stent before the anterior closure. This allows a leak test to be performed after completion of the anastomosis

models still come at a relative expense to the trainees both financially and ethically.

To enhance accessibility of surgical simulation in the resource-poor setting, we have developed this very-lowcost, easily reproducible series of models. Beyond basic surgical instruments, our models require animal tissue which would be accessible within the locality of the healthcare system. For these reasons, it is believed the invaluable surgical techniques-technical and cognitive skills-learned from each model outweigh the low-fidelity nature of the simulations.

\section{Conclusion}

These models provide vital access to paediatric surgeons (in high-, middle- and low-income settings) and allow them a safe platform to learn technical, non-technical and cognitive skills that can be developed and eventually transferred into a surgical setting with improved clinical and patient-centred outcomes.

Acknowledgements Special thanks go to the International Affairs Committee of the British Association of Paediatric Surgeons. All images are copyright of Department of Medical Illustration, University Hospitals of Leicester and used with permission.

Open Access This article is distributed under the terms of the Creative Commons Attribution 4.0 International License (http://crea tivecommons.org/licenses/by/4.0/), which permits unrestricted use, distribution, and reproduction in any medium, provided you give appropriate credit to the original author(s) and the source, provide a link to the Creative Commons license, and indicate if changes were made.

\section{References}

1. Meara JG, Leather AJ, Hagander L et al (2016) Global Surgery 2030: evidence and solutions for achieving health, welfare, and economic development. Int J Obstet Anesth 25:75-78

2. Barsness K (2014) Simulation-based education and performance assessments for pediatric surgeons. Eur J Pediatr Surg 24(4):303-307

3. Cook DA, Brydges R, Hamstra SJ et al (2012) Comparative effectiveness of technology-enhanced simulation versus other instructional methods: a systematic review and meta-analysis. Simul Healthc 7(5):308-320

4. Sanchez-Margallo FM, Rivera Barreno R, Staines-Orozco H et al (2017) A novel simulation system for thoracoscopic congenital diaphragmatic hernia correction. Surg Endosc Other Interv Tech 31(2(1)):S59

5. Simforoosh N, Khazaeli M, Nouralizadeh A et al (2011) Laparoscopic animal surgery for training without sacrificing animals; introducing the rabbit as a model for infantile laparoscopy. J Laparoendosc Adv Surg Tech A 21(10):929-933

Publisher's Note Springer Nature remains neutral with regard to jurisdictional claims in published maps and institutional affiliations. 